\title{
Riboswitching off bacterial growth
}

DOI:

10.1038/nrmicro1603

URLs

Bacillus subtilis

http://www.ncbi.nlm.nih.gov/ entrez/query.fcgi?db=genome $\operatorname{prj} \& \mathrm{cmd}=$ RetrieveEdopt $=$ Over viewElist_uids=17579
Current antibiotics target just four cellular processes, and bacteria have found ways to compensate for them all. New drugs with novel mechanisms of action are therefore urgently needed. But identifying drug targets that are essential for bacterial survival, are conserved across species and that lack a human homologue is a significant challenge.

Thankfully it seems that bacteria still have some essential survival pathways that we have not yet exploited. Writing in Nature Chemical Biology, Blount et al. provide evidence that bacterial riboswitches - RNA structures that function as metabolic sensors and regulate gene expression accordingly - could represent one such novel class of antibiotic target.

Riboswitches are found in the

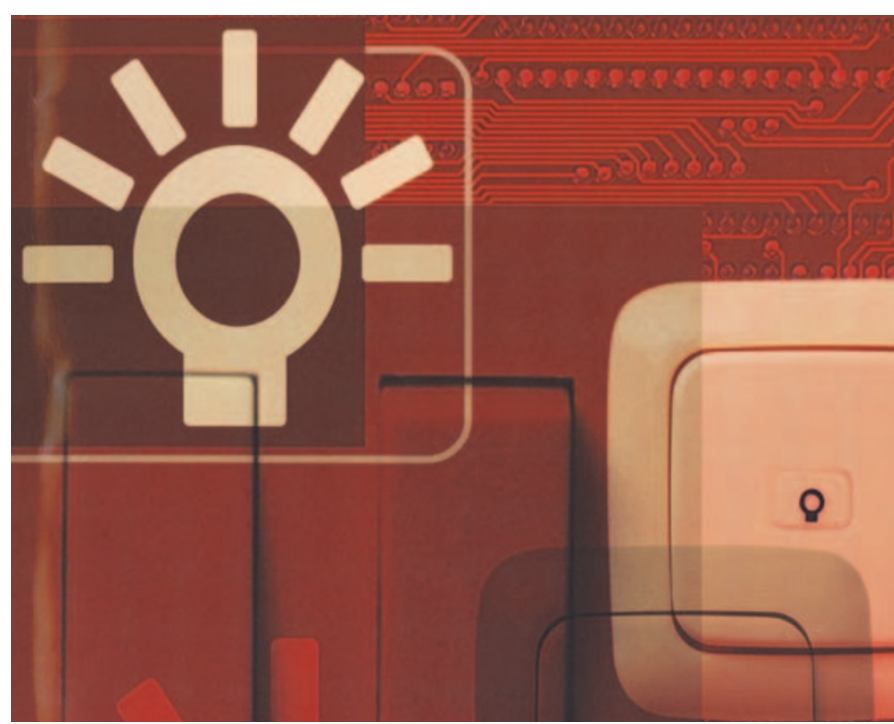

$5^{\prime}$-untranslated regions of bacterial mRNAs and form receptors that bind specific metabolites, leading to the expression of genes involved in metabolite biosynthesis and transport. Blount et al. proposed that blocking riboswitch binding could halt the expression of these essential genes.

Lysine riboswitches are found in many species of bacteria and are known to downregulate two important proteins: a lysine-specific importer and aspartokinase II, the initiating enzyme in a biosynthetic pathway that generates precursors of cell-wall biosynthesis and spore formation. The authors therefore designed a series of lysine analogues aimed at inhibiting lysine riboswitches and interfering with this pathway.

The aim was to find lysine analogues that showed specificity for the lysine riboswitch but were sufficiently chemically different to lysine itself that they did not interact with host enzymes or function as a nutritional source for the bacterium. Twelve analogues were tested for their capacity to bind to the lysine riboswitch of Bacillus subtilis. The different specificities attributed to various analogues provided useful insights into the mechanism of riboswitch-ligand binding and identified molecular features that are essential for ligand recognition.

Having established that the analogues bind to the riboswitch, the authors then studied whether they could inhibit bacterial growth.
In in vitro cultures of $B$. subtilis, five of the analogues (tested at $100 \mu \mathrm{M}$ concentrations) slowed bacterial growth, and three of these analogues completely inhibited bacterial growth for 24 hours. A reporter-gene assay measuring $\beta$-galactosidase expression under control of the lys $C$ riboswitch in B. subtilis showed that the analogues exert their effects by repressing lys $C$ at concentrations similar to the minimal inhibitory concentrations required to inhibit bacterial growth.

Strains of $B$. subtilis that were cultivated to be resistant to one of the analogues were found to have a single point mutation in the lys $C$ riboswitch, but not in the $y v s H$ gene that encodes the lysine transporter. This, and the observation that mutation of the riboswitch conferred resistance to other antibacterial lysine analogues, confirms that the antibacterial activity of the analogues can be attributed to lysine riboswitch-mediated repression of aspartokinase II.

The authors warn that compensatory mechanisms might exist that will enable bacteria to glean lysine from the host. However, this initial study certainly acts as a proof of principle for the strategy of targeting riboswitches and warrants further investigation into the development of novel antibacterial drugs.

Joanna Owens, Senior Editor, Nature Reviews Drug Discovery

ORIGINAL RESEARCH PAPER Blount, K. F. et al. Antibacterial lysine analogs that target lysine riboswitches. Nature Chem. Biol. 3, 44-49 (2007). 